\title{
A Survey on Blurred Images with Restoration and Transformation Techniques
}

\author{
Rohina Ansari \\ Department of Computer Science \\ RITS, Bhopal
}

\author{
Himanshu Yadav \\ Department of Computer Science \\ RITS, Bhopal
}

\author{
Anurag Jain \\ Department of Computer Science \\ RITS, Bhopal
}

\begin{abstract}
In modern science and technology, digital images gaining popularity due to increasing requirement in many fields like medical research, astronomy, remote sensing, graphical use etc. Therefore, the quality of images matters in such fields. There are many ways by which the quality of images can be improved. Image restoration is one of the emerging methodologies among various existing techniques. Image restoration is the process of simply obtaining an estimated original image from the blurred, degraded or corrupted image. The primary goal of the image restoration is the original image is recovered from degraded or blurred image. This paper contains the review of many different schemes of image restoration that are based on blind and nonblind de-convolution algorithm using transformation techniques.
\end{abstract}

Keywords- Image processing, Blurred images, Padding, kernel, Canny edge, Transformation techniques.

\section{INTRODUCTION}

Image processing is an emerging field of practical study of images [1]. In this area image can analyze in order to enhance the quality, decrease the size and if the image is distorted than restore the image. Image restoration is a process of recovering an original image from the observed image that can be degraded or blurred or corrupted noisy image. The basic idea of image restoration is obtaining an image that is free from blur and noise from the observed image in order to improve the image quality. There are number of factors that are responsible for blurring in image like shaky camera or motion blur, atmospheric reasons, motion between camera and original image and bad focus camera[4]. When the intensity of an observed point image is spread over the several pixels, image goes blurred or a flat color image occurs. The point is called Point Spread Function (PSF) also known as blurring operator. Image de-blurring is a method of removing or minimizing blur from the degraded image observation [5]. On the basis of blurring parameter, image deblurring is divided into two classes, one is blind and other one is non-blind. In the first class, blurring operator is unknown while in the second class blurring operator is known. However in real applications, the information about PSF is not known. As a result, blur detection must be performed on image in addition to image restoration. The difficulty of image de-blurring is to recover high frequency information from blurred image. Image can be blurred or degraded using low -pass filter and its noise. There are many low -pass filter available like Gaussian filter, Salt or pepper filter, Speckle filter [2,5].

\section{IMAGE PROCESSING}

An image is composed of sub-images called as Region of Interest (ROI) or regions. So an image contains a collection of objects act as a base of these regions. It can happen that image processing is performed on selected regions [1].In image processing, it is mostly required that an image should be in digitized form. Before going to process an image it is firstly converted into digital form. Digitization involves sampling and quantization .After converting an image into bit information, processing is performed. There are three main techniques of image processing includes: image enhancement, image restoration and image compression [2].

\subsection{Image enhancement:}

It concerns with the quality of the image by using knowledge of human visual system responses to improve an image visually.

\subsection{Image compression:}

It involves reducing the massive amount of data needed to represent an image.

\subsection{Image Restoration:}

It is concerns with the quality of image recovering an original image from distorted image.

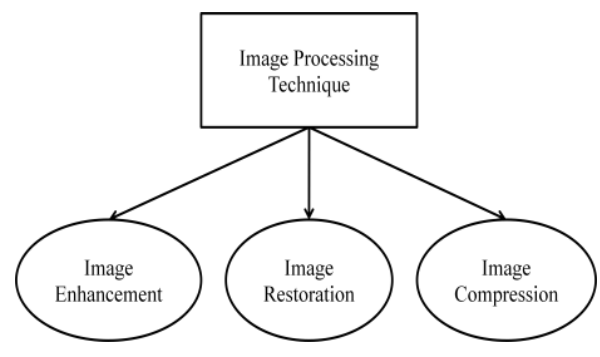

Fig.1. Image processing techniques

According to Ms.S.Ramya [6] an efficient approach of image restoration of blurred images is introduced using Canny edge detection method for detecting edges in the image Gaussian filter is used as a low pass filter to blur an image. This method is based on Blind deconvolution. There are basically two methods for image restoration that are blind and non blind. The blind deconvolution is more complex than non-blind but produces good result as compared to the non-blind deconvolution method. The technique uses Gaussian filter as low pass filter and ringing effect is produced using Canny edge detector and blur is removed in the image. Sun qi proposed an efficient method for the blurred image restoration using Maximum Likelihood [3]. This generates the effective results.

An adaptive Blind Image restoration algorithm for corrupted and degraded images has been proposed in [4]. Here the blur type is checked on the basis of PSF and after that image restoration is performed according to the type of blur detected using Wiener filter if the blur type is usual and uses NAS-RIF algorithm for 
other type of blur. The usual type of blur include Gaussian blur, defocus blur, motion blur.

Jinlian Zhuang and Youshen Xia proposed a new method for image restoration using L1 regularization approach [5]. Here in this work corrupted pixel is detected using L1 regularized cost function and then NAS-RIF algorithm is used to filter these corrupted pixels. The algorithm implemented here produces good results in less computation time as compared to the other blind de-convolution algorithms.

Another new approach is introduces for image restoration in [8]. Here the concept of multi channel blind separation of images is used for the analysis of documents. The basic idea behind this method is to recover clean maps the main fore grounded text. Enhancement and extraction of other document also analyzed .The corrupted pixel are estimated using Bayesian approach and Gibbs a prior is used for filtration.

Ryu NAGAYASU has proposed a new technique of restoring images using Kalman filter [9].To get the best quality of image from noisy blurred image is the main task of this method. This is done by using the auto regressive in the Kalman filter technique. Basically the Kalman filter removes the boundary areas of the degraded image. This method is feasible to use. Renting Liu Jiaya Jia has proposed a new technique of blind deconvolution for reducing the boundary artifacts [10]. So the original image is recovered from the degraded image. In this method, the blur is removed on the basis of PSF. A PSF is a point spread function and defined as a point in the observed image whose intensity is spread over the several pixels. With the help of PSF knowledge, the blur level of the pixel is detect in the blurred image and then filtration is applied on the image using Fast Fourier Transformation (FFT) to filter the particular blurred region of the image that was detected using PSF. So the degradation of the image is reduced.

Jong-Ho Lee, Yo-Sung Ho proposed a new technique for image restoration using non-blind deconvolution [11]. In non-blind deconvolution of image restoration, the blurring parameter or PSF is known. On the basis of prior knowledge of PSF the original image is recovered from the blurred/degraded image. The details of image are preserved. FFT is used for filtration to get the effective results.

\section{BLUR an IMAGE}

A linear degradation model is used to degrade/blur an image. In the model, first original image is loaded and Gaussian filter and its additive noise is applied to completely blur the image as a low pass filter [4]. The model shown in figure. 2

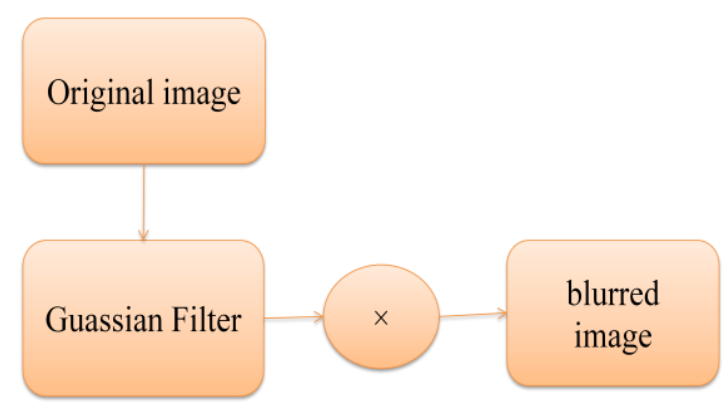

Fig.2. Degradation model to blur an image
Image Deconvolution is a linear image restoration difficulty in which the parameters of the actual image are estimated using the experiential or degraded image and a known Point Spread Function (PSF). Blind Image Deconvolution is a more difficult where image recovery is performed with slight or no earlier information of the PSF. The benefits of Deconvolution are superior resolution and enhanced quality [5, 6, 7].

An adaptive blind image restoration provides an automatic recognition system for degraded images. This involves the two main steps are:

1. First of all, classified the types of blur.

2. After that in second step, image restoration will perform according to blur type.

There are two types of blur is classified usual or others. The usual includes motion blur, Gaussian blur, defocus blur. If the blur type is usual then PSF can be estimated with corresponding blur identification method and restored the image with Wiener filter. But for the other type blur, NAS-RIF blind restoration Algorithm is used. Thus, for several degraded images an adaptive blind restoration is implemented. It improves the quality of restored images [5]. The adaptive blind image restoration algorithm principle is showed below.

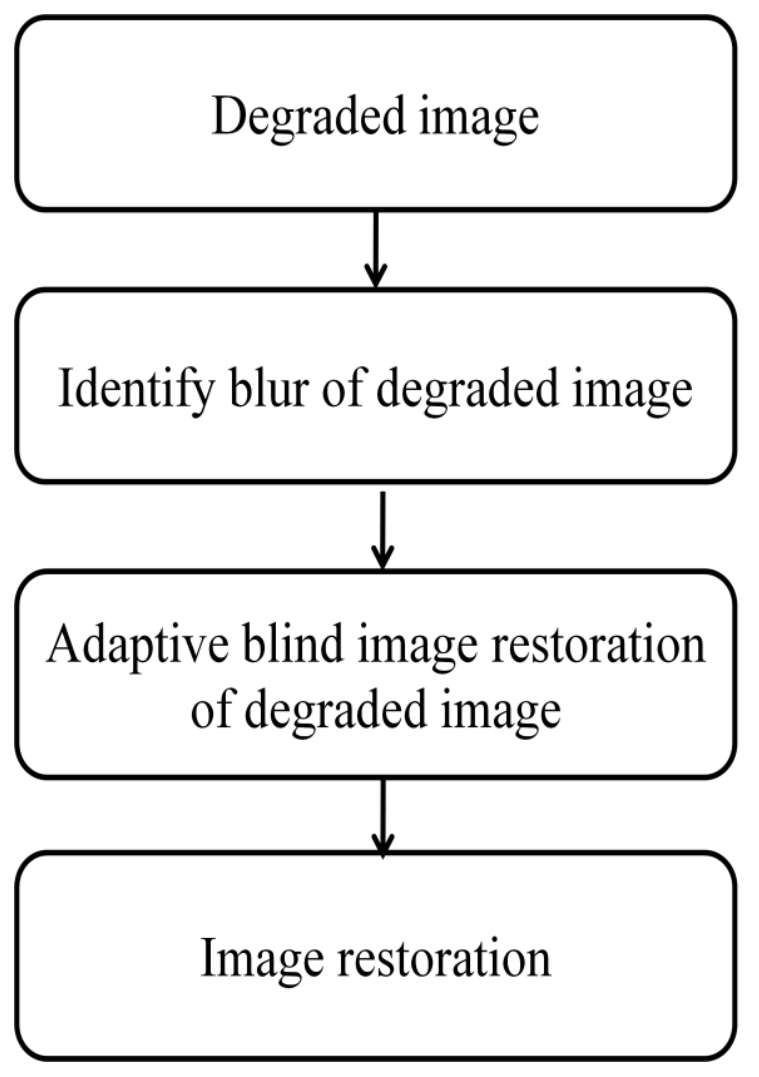

Fig.3. The basic principle of adaptive blind image restoration.

\section{BLUR DETECTION WITH EDGE}

In an image different types of edges are present. An edge is classified into three classes: Dirac-Structure, Step-Structure [3].Step is further classified into Astep-Structure on the basis of intensity variation. The diagrammatical description of edges is 
shown in figure $4[10,11,12]$. When the blur occurs, DiracStructure and Astep-Structure edge disappear in both motion blur and out-of-focus blur and both Roof-Structure and GstepStructure becoming lose their sharpness. The sharpness is measured by a parameter called the sharpness parameter. This method detects whether a given image is blurring or not on the basis of Dirac-structure and Astep-Structure .If blur, then blur extent is identified by taking sharpness into account of Roofstructure and Gstep-Structure.

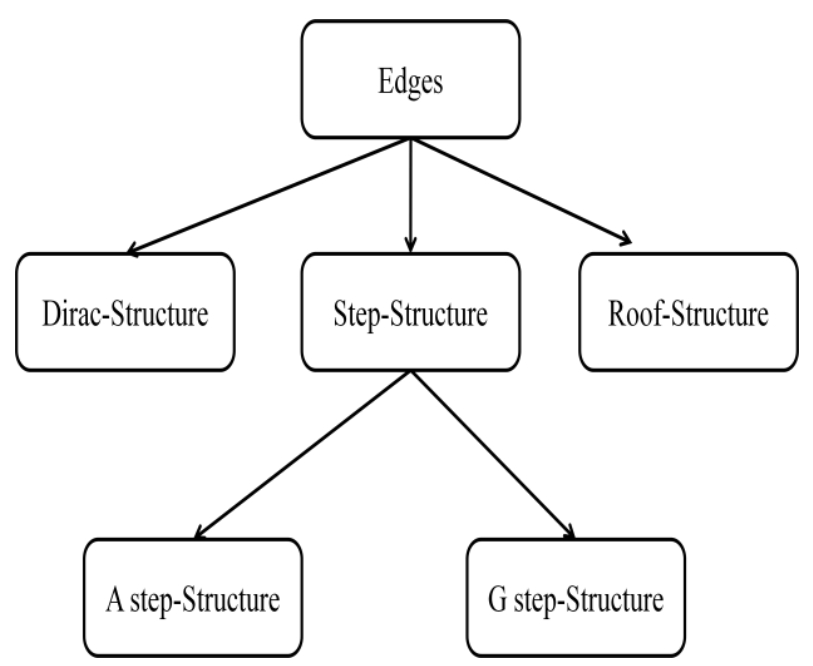

Fig.4. Classification of edges.

\subsection{Canny Edge Detection and Ringing Effect}

J.Canny proposed an optimal edge detection method. It detects edges in images using multi-Stage procedure. According to this algorithm the, following criteria should be satisfied [3]:

- There should be low probability of both missing true edges and false edge detection.

- There should be minimum distance between the detected edge and true edge in the image.

- For each edge in the image, only one mark should be allowed.

In this algorithm, firstly the image is smoothen/blur using Gaussians filter to reduce the noise. After this, find the local gradient $\mathrm{W}(\mathrm{x}, \mathrm{y})$ and edge direction computed at each point to highlight the high derivative regions .Since the edge in image may point various direction like horizontal, vertical and diagonal, Canny algorithm uses four filters to smoothen the image. Two for horizontal and vertical edges and other two for two diagonal edges [10].The edge direction operator return a first derivative value in horizontal and vertical direction. The edge direction and gradient may be given as

$$
\begin{gathered}
\mathrm{H}=\sqrt{\mathrm{W}_{\mathrm{x}}^{2}}+\sqrt{\mathrm{W}_{\mathrm{y}}^{2}} \\
\theta=\arctan \frac{\mathrm{W}_{\mathrm{y}}}{\mathrm{W}_{\mathrm{x}}}
\end{gathered}
$$

The derivative region are found to suppress any pixel that is not at the maximum( non-maximum suppression).The pixels are then Threshold M1 and M2 with M1<M2.If the magnitude of certain pixel less than M1 then it is set to zero, if greater than M2 then it is made an edge. And if the magnitude of pixel is between M1 and $\mathrm{M} 2$, then it is set to zero unless there is a path from this pixel to a pixel with gradient above M2.

\subsubsection{Edge tapper for ringing effect}

Edge tapper is a function which is used to avoid the ringing effect. Before the image is passing to the blurring function, it first passes to Edge tapper for preprocessing. It first blur the entire image and then replacing the center pixel of blurred image with the original image in order to remove high intensity drop-off at the edges. [10]

\section{PADDING THE KERNEL OF IMAGE}

A convolution kernel is a matrix that describes the contribution of pixel to the pixel located in the original or final image [17, 18]. Convolution is a process of operating a function on another one which is called kernel .The used kernel depends on the required effect .Example of padding using the kernel are shown below.

Example:
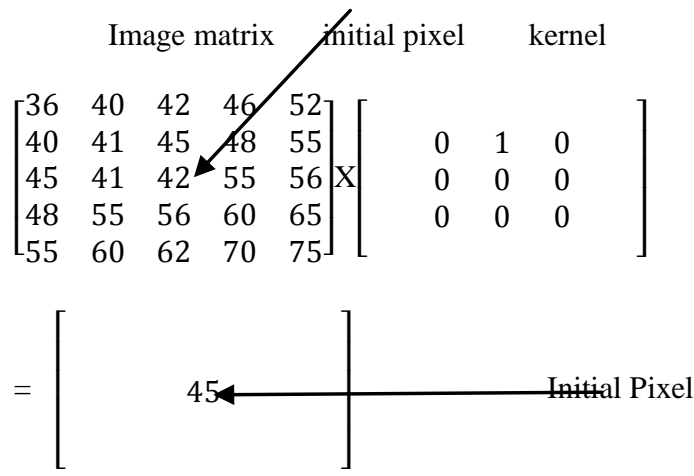

In the above example all pixel of the kernel area read successively, from top to bottom and left to right. It multiplies the values of each pixel by kernel corresponding value and adds to the results. The initial pixel has become 45 :

$[(41 \times 0)+(45 \times 1)+(48 \times 0)+(41 \times 0)+(42 \times 0)+(55 \times 0)+$ $(55 \times 0)+(56 \times 0)+(60 \times 0)]=45$

Here initial pixel is moved a pixel downward i.e. 45 takes the location of 42 in the resulting image matrix.

Steps for padding the image. The process of adding new pixels in the image for filtering purpose and for annotations or any remarks is called padding the image. The process of padding the image is listed below in figure.5. 


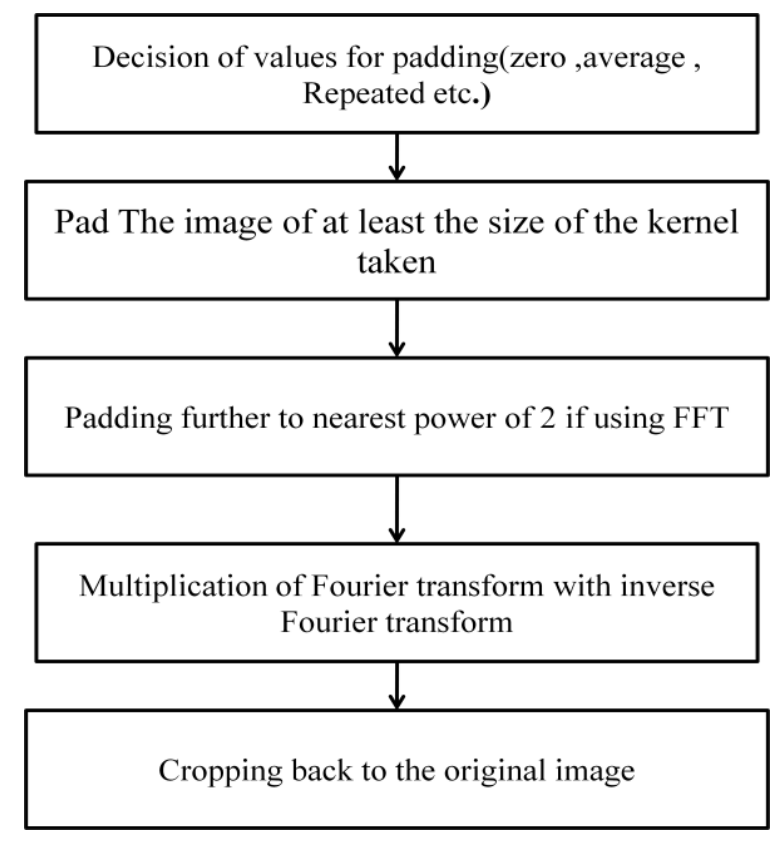

Fig.5. Steps for padding the image.

\section{TRANSFORMATION TECHNIQUES}

Transformations are used for image filtering, to remove noise, to sharpen or to extract information from the image. An image may be represented in the both spatial and frequency or Fourier domain. Pixel value and location in image are in three dimensions i.e. $\mathrm{x}, \mathrm{y}$ and $\mathrm{z}$. $\mathrm{x}$ refer to column, $\mathrm{y}$ refer to rows and $\mathrm{z}$ refers to the value in spatial domain. On the other hand in frequency domain, the value and location in image depends upon the frequency of a pixel within image [15]. Pixel location is represented by its $\mathrm{x}$ and $\mathrm{y}$ frequencies and its value is represented by an amplitude. However a pixel value ant location may also be represented in time-frequency or Wavelet domain. In frequency or Fourier domain an image is partially transform while in Wavelet domain image is fully transformed. In spatial domain, some process performed may be very computationally expensive .But after transforming an image; these same processes can be easily performed. FFT is used to transform an image spatial and frequency domain. The FFT decomposes the image into sine and cosine of changing phase and amplitude. The resulting transformed values represent the horizontal and vertical frequency amplitude [16]. Gradual Changes are represented by low frequency while high frequency represents abrupt changes in the image. Low frequencies try to contain information as more as possible as they represent entire shape of the image. High frequency provides details about the image but they contain noise. In the frequency domain, lowest frequencies contain most of the information, which is represented by the large peak value in the centre of the data. In case of transformation of image, lowest frequency peak is composed of brightest pixel. To convert the image into spatial domain from the frequency domain Inverse transformation is needed called Inverse FFT.

An image is partially converted into frequency domain in some transformations, Time-frequency or Wavelet domain is the most common .The FFT decomposes an image with sine and cosine while in Discrete Wavelet Transform(DWT) [16] is used to transforming the image from spatial to time-frequency domain and back again. The Wavelet functions can be applied multiple times the image portion. For removing noise image can be altered by using frequency domain.

Wavelet Transform is good known for its multi-resolution analysis ability. Discrete Wavelet transform (DWT) performs fast computation. Another property of DWT is that it is easily implemented have less computation time and resource required $[12,13,14]$. DWT provides a time scale representation of digital signals by using filtering techniques. The DWT is calculated with successive low pass and high pass filtering of discrete time domain signal. The low pass filter produces coarse approximation while high pass filter produces detail information. Using DWT, the frequency resolution becomes good at low frequency while at high frequency, time resolution becomes good. The DWT gaining popularity due to its reducing distortion ability in reconstructed signal.

\subsection{Haar wavelets}

Haar belongs to the group of DWT. Haar Wavelet transform (HWT) is the simplest wavelet. It has a property that it s not differentiable and it is used for fast calculation [15].

The scaling function $\phi(t)$ of Haar is expressed as

$$
\phi(\mathrm{t})= \begin{cases}1, & 0 \leq t<1 \\ 0, & \text { otherwise }\end{cases}
$$

Haar matrix: The $2 \times 2$ Haar matrix is

$$
\mathrm{H}_{2}=\left[\begin{array}{cc}
1 & 1 \\
1 & -1
\end{array}\right]
$$

Haar transform is derived from Haar matrix and Haar transformation matrix is expressed as

$\mathrm{H} 4=\left[\begin{array}{cccc}1 & 1 & 1 & 1 \\ 1 & 1 & -1 & -1 \\ 1 & -1 & 0 & 0 \\ 0 & 0 & 1 & -1\end{array}\right]$

\subsubsection{Discrete Haar function}

The Discrete Haar function may be the function which is determined by sampling the Haar function at The Discrete Haar function may be the function which is determined by sampling the Haar function at $2^{\mathrm{n}}$ points. This function can be expressed in the form of matrix. The matrix is denoted by $\mathrm{H}(\mathrm{n})$ and each row of the matrix has discrete Haar sequence Haar $(w, t)$ or discrete Haar function [16] .In Haar (w,t), w specifies the number of Haar function and $t$ specifies the interval. The Haar function of any direction may be expressed with the help of recurrence relation:

$$
H(n)=\left[\begin{array}{ccc}
H(n-1) & \times 1 & 1 \\
2^{(n-1) / 2} I(n-1) & \times 1 & -1
\end{array}\right]
$$

$\mathrm{H}(0)=1$ and $\mathrm{H}(\mathrm{n}) \neq \mathrm{H}(\mathrm{n})^{\mathrm{T}}$ for $\mathrm{n}>1$ and $\mathrm{H}(\mathrm{n})^{-1}=2^{-\mathrm{n}} \cdot \mathrm{H}(\mathrm{n})^{\mathrm{T}}$,

Where $\mathrm{I}$ is the Identity matrix and $\times$ is the Dirac delta product.

\section{CONCLUSION}

Digital Image processing is an emerging field now days. There are number of challenges like, compression, visualization, reorganization, brightness preserving, image restoration and many more. In spite of having all these issues some time noise is a still major problem in image processing. Restoration is a technique of image processing in which the quality of image is improved by obtaining the noise free or degradation free image. This task is accomplished with the help of various deconvolution techniques including blind and non-blind. Whether, Transformation and filtration techniques also used for fast computation and quality improvement. It seems to be that there is a need to remove the noise from image in order to de-blur the image. 


\section{ACKNOWLEDGMENT}

I would like to say thanks to my HOD "Prof. Anurag Jain" and guide "Mr. Himanshu Yadav" who gives their knowledge and time in order to complete this paper. This paper will never complete without the support of faculty member CSE department of Radharaman Institute of technology and science Bhopal.

\section{REFERENCES}

[1]. Syaidatul Akma Mohd Zuki, Ihsan Mohd Yassin, "A review of image processing technique in particle mixing analysis", IEEE 2012, pp 466-469.

[2]. M. Ravudu, V. Jain, and M.M.R. Kunda, "Review of image processing techniques for automatic detection of eye diseases ", IEEE 2012, pp 320-325.

[3]. You-Yi Zheng, Ji-Lai Rao and Lei Wu, "Edge detection methods in digital image processing", IEEE 2010, pp 417473 .

[4]. BI Xiao-jun, WANG Ting," Adaptive Blind Image Restoration Algorithm of Degraded Image”, IEEE 2008.

[5]. Jinlian Zhuang and Youshen Xia "A Two-Dimensional Iterative Algorithm for Blind Image Restoration based on An L1 Regularization Approach", 2010 3rd International Congress on Image and Signal Processing (CISP2010), pp. $51-55,2010$.

[6]. S.Ramya, T.Mercy Christial "Restoration of blurred images using Blind Deconvolution Algorithm", 2011 International Conference on Emerging Trends in Electrical and Computer Technology (ICETECT), pp. 496 - 499, March 2011.

[7]. Sun qi1, Hongzhi Wang 2, Lu wei3," An iterative blind deconvolution image restoration algorithm based on adaptive selection of regularization parameter",IEEE 2009.

[8]. Anna Tonazzini, Ivan Gerace, and Francesca Martinelli," Multichannel Blind Separation and Deconvolution of Images for Document Analysis”, IEEE 2010.
[9]. Ryu Nagayasu, Naoto Hosoda, Nari Tanabe, Hideaki Matsue, Toshihiro Furukawa," Restoration Method For Degraded Images Using Two-Dimensional Block Kalman Filter With Colored Driving Source”,Ieee 2011.

[10].Renting Liu Jiaya Jia," Reducing Boundary Artifacts In Image Deconvolution,"IEEE 2008

[11].Jong-Ho Lee, Yo-Sung Ho, "High-quality non-blind image deconvolution with adaptive regularization", Elsevier 2011.

[12].Y.Y. Tang, and L. Feng, "Multi-resolution Adaptive Wavelet Edge Detection," The 2th International Conference on Multimodal Interface, January 1999, v7-v11.

[13].Y.Y. Tang, and L. Yang, "Characterization and Detection of Edges by Lipchitz Exponents and MASW Wavelet Transform," 14th International Conference on Pattern Recognition, August 1998, pp. 1572-1574.

[14].Y.Y. Tang, L. Yang and J. Liu, "Characterization of DiracStructure Edges with Wavelet Transform," IEEE Trans. Systems, Man and Cybernetics, Part B, February 2000, pp.93-109.

[15].Wangli Ouyang, Rengi Zhang, Wai-Kuen K. Cham, "Fast pattern matching using orthogonal Haar transform". IEEE 2010, pp 3050-3057

[16].Dimitrios A.Karras,"Efficient medical image compression/reconstruction applying Discrete Wavelet Transform on texturally clustered regions", IEEE 2005,pp 87-91(dwt)

[17].Sunghyun Chao, Yasuyuki Matushita,Seung-Yong Lee, "Removing Non-uniform Motion Blur from images", IEEE 2007,pp 1-8.

[18].Hideyuki Imai, Masaaki Miyakoshi, "Image restoration with kernel component estimation in singular observation process", IEEE 2003, pp 186-189. 